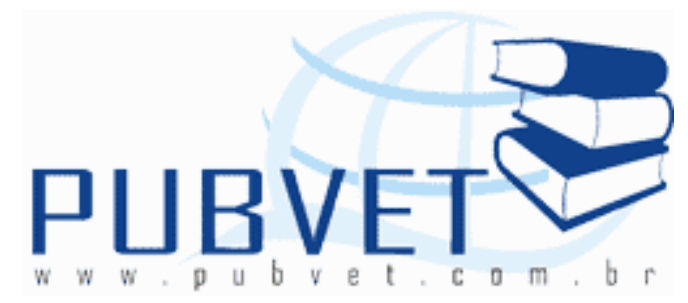

PUBVET, Publicações em Medicina Veterinária e Zootecnia.

\title{
As inovações tecnológicas na ovinocultura brasileira e seus efeitos na organização do sistema agroindustrial
}

Camila Raineri ${ }^{1}$, Mariana Rosário Freitas Lopes ${ }^{1}$, Thayla Sara Soares Stivari ${ }^{1}$, Carina Simionato de Barros ${ }^{1}$, Bruno Prosdocimi Nunes ${ }^{2}$, Augusto Hauber Gameiro $^{3}$

1 Pós Graduação em Nutrição e Produção Animal da Faculdade de Medicina Veterinária e Zootecnia. Departamento de Nutrição e Produção Animal. Universidade de São Paulo.

${ }^{2}$ Zootecnista, mestre em Qualidade e Produtividade Animal pela Faculdade de Zootecnia e Engenharia de Alimentos. Universidade de São Paulo.

${ }^{3}$ Professor do Departamento de Nutrição e Produção Animal da Faculdade de Medicina Veterinária e Zootecnia da Universidade de São Paulo. Coordenador do Laboratório de Análises Socioeconômicas e Ciência Animal (LAE/FMVZ/USP).

\section{Resumo}

A ovinocultura brasileira vem sofrendo pelo menos duas grandes mudanças: a mudança de foco, da produção de lã para a de carne; e sua expansão para novas áreas. Isto gerou um vácuo de informações aplicáveis a animais e condições distintos. Pesquisas se iniciaram e novas tecnologias passaram a ser desenvolvidas e aplicadas para subsidiar a atividade. Este trabalho analisou a influência das inovações tecnológicas sobre a organização do sistema agroindustrial (SAG) ovino, sob a ótica da Economia dos Custos de Transação 
(ECT). Identificou-se as principais inovações tecnológicas observadas nas áreas de i) produção, ii) processamento e iii) gestão. Elas tenderam a reduzir o impacto dos pressupostos comportamentais descritos pela teoria da ECT: racionalidade limitada e oportunismo, embora talvez este efeito ainda não possa ser verificado na prática. Já os atributos das transações - especificidade e freqüência - passam a ganhar mais destaque. O terceiro atributo das transações, a incerteza, tende a ser reduzido, embora algumas inovações tenham o efeito oposto. As alterações nos atributos demonstram que as inovações tecnológicas foram capazes de condicionar a estruturação e organização do SAG da carne ovina. Outras evidências são o surgimento de novos atores, e a instalação de novas formas de governança.

Palavras-chave: cordeiro, custos de transação, governança, inovação, tecnologia

\title{
Technological innovations in Brazilian sheep industry and its effects on supply chain organization
}

\begin{abstract}
Brazilian sheep industry has been through at least two deep changes: the change of focus, from wool to meat production; and its expansion to new areas. That revealed a lack of knowledge applicable to animals of different genotypes, and to areas with conditions that were very distinct from those in the south region. Many researches started and new technologies began to be developed and applied to support farmers. This paper studied the effect of technological innovations on lamb supply chain organization from the viewpoint of Transaction Costs Economy Theory (TCE). The main technological innovations observed in the following areas were identified: i) production, ii) processing e iii) management. The innovations tended to reduce the impact of the behavioral assumptions described by the theory of TCE, limited rationality and opportunism, although that effect cannot be actually verified. The transaction attributes - specificity and frequency - received more importance.
\end{abstract}



2013.

The third transaction attribute - uncertainty - tends to be reduced, although some innovations have the opposite effect. These impacts on the attributes demonstrate that technological innovations were capable of conditioning the organization of Brazilian lamb supply chain. Other evidences are the appearance of new players, and the occurrence of new governance forms.

Keywords: governance, innovation, lamb, technology, transaction costs

\section{INTRODUÇÃO}

A ovinocultura está presente em todos os estados brasileiros, em virtude de sua adaptabilidade às condições adversas e de sua habilidade para transformar material fibroso e de baixo valor nutritivo em alimentos de alto valor protéico. A carne desses animais, em particular, representa a mais importante fonte de proteínas para uma grande parcela de pequenos agricultores e habitantes das pequenas cidades do Nordeste e da Região Sul (JESUS JUNIOR et al., 2010). Dos ovinos, é possível explorar a carne, o couro (em menores volumes) e a lã. Também há uma pequena produção de leite, principalmente para a fabricação de queijos especiais.

Viana (2008) afirma ter sido no início do século XX que a produção de ovinos se tornou uma atividade econômica no Brasil, mais especificamente no estado do Rio Grande do Sul. Segundo Bofill (1996), com a deflagração da Primeira Guerra Mundial, em 1914, houve entrada do Rio Grande do Sul no mercado dos países em conflitos, com a procura e conseqüente ascensão de preços da carne e da lã. Durante muitos anos o foco da ovinocultura foi a produção de lã, desenvolvendo-se nos estados da região sul.

No final da década de 1990, uma nova face da atividade passou a se desenvolver em outras regiões brasileiras. A produção de carne ovina é uma atividade que vem se desenvolvendo gradativamente no país desde então, mudando o foco e crescendo em regiões onde antes a ovinocultura era insignificante, viabilizando sistemas de produção animal em pequenas 

2013.

propriedades e tornando-se mais uma alternativa de investimento no meio agropecuário.

O Brasil conta, segundo a Pesquisa Pecuária Municipal de 2009, com quase 17 milhões de ovinos. A maior parte destes animais é destinada à produção de carne, cujos maiores consumidores são o estado de São Paulo, que tem renda superior aos demais e abriga grandes colônias árabes e nordestinas, e a Região Nordeste. Apesar de apresentar baixo consumo relativo, a produção nacional é insuficiente e o Brasil importa ovinos vivos, carcaças congeladas e carne desossada, basicamente do Uruguai, para suprir o consumo interno (JESUS JUNIOR et al., 2010).

Pela falta de tradição e experiência da maior parte dos novos ovinocultores das regiões Sudeste, Centro-Oeste e Norte, bem como pela escassez de subsídios científicos para a condução da atividade, muitas pesquisas passaram a ser realizadas a partir do início dos anos 2000 . Um grande vácuo de informações sobre nutrição, manejo, pastagens, sanidade, genética, comportamento e outras áreas do conhecimento precisava ser preenchido. E mais: tais conhecimentos precisavam ser aplicáveis a ovinos de corte, e a regiões com condições edafoclimáticas e sócio-econômicas muito distintas das do sul do país, onde havia a maior gama de informações disponíveis. Neste contexto, novas tecnologias passaram a ser desenvolvidas com o objetivo de incrementar a produtividade do rebanho nordestino e de fornecer subsídios para as novas criações.

Resende et al. (2010) destacam que na última década foi observado um aumento de $41 \%$ nas publicações mundiais envolvendo pequenos ruminantes, o que foi acompanhado por aumento ainda mais expressivo, $219 \%$, em âmbito nacional. A evolução também foi observada sob os aspectos metodológicos dos projetos, ressaltando a abordagem multidisciplinar nas pesquisas mais atuais. Nos últimos dez anos, houve crescimento do aporte de recursos para editais temáticos demandados pela sociedade e pela cadeia da carne e do leite, além da preocupação com qualidade, competitividade e sustentabilidade. 

2013.

Apesar da maior parte de tais pesquisas terem se iniciado há poucos anos, e portanto de não serem tão abundantes quanto em outras espécies, diversos avanços vêm sendo conquistados em diferentes campos. De acordo com Resende et al. (2008), não somente o efetivo do rebanho nacional tem aumentado, mas sua capacidade produtiva também tem evoluído. Para tal avanço, merecem destaque os aspectos do melhoramento genético visando a produção de carne e leite, na nutrição, da sanidade, entre outros (SEBRAE, 2005).

No entanto, Morand-Fehr e Boyazoglu (2008), citados por Resende et al. (2010), afirmam que apesar de existirem vários artigos de boa qualidade científica, muitas vezes estes conhecimentos não chegam a grande parte do setor produtivo. Há, portanto a necessidade de se investir também em comunicação e extensão, já que a baixa produtividade da ovinocultura ainda é a realidade mais presente em diversas regiões brasileiras, embora algumas mudanças ao longo dos elos da cadeia tenham sido observadas.

O objetivo deste trabalho é analisar e demonstrar como as inovações tecnológicas na produção de ovinos influenciaram a organização do sistema agroindustrial, sob a ótica da Economia dos Custos de Transação (WILLIAMSON, 1979).

\section{MATERIAL E MÉTODO}

O presente estudo foi conduzido a partir de metodologia exploratória e descritiva (VIANA, 2008). Realizou-se uma revisão da literatura disponível de forma a caracterizar a evolução da ovinocultura no Brasil e identificar as principais inovações tecnológicas observadas no último século. A pesquisa envolveu tecnologias aplicadas a três grandes áreas do sistema agroindustrial ovino, sendo i) produção, ii) abate e processamento e iii) gestão.

A partir dos dados coletados, a Economia dos Custos de Transação (WILLIAMSON, 1979) foi utilizada para demonstrar as modificações estruturais e organizacionais sofridas pelo sistema agroindustrial ovino brasileiro em 
função das inovações tecnológicas. Desta forma, as inovações tecnológicas são tratadas como condicionantes da formação das estruturas de governança da cadeia ovina.

Explorou-se o impacto das inovações sobre os pressupostos comportamentais e atributos das transações descritos pela ECT. Os pressupostos comportamentais apresentados pela teoria são i) racionalidade limitada e ii) oportunismo; e os atributos das transações são i) especificidade dos ativos, ii) incerteza e iii) freqüência.

\section{RESULTADOS E DISCUSSÃO}

As informações coletadas a respeito das principais inovações tecnológicas ocorridas no sistema agroindustrial ovino nas últimas décadas encontram-se relacionadas a seguir.

\subsection{INOVAÇÕES NA ÁREA DA PRODUÇÃO}

\subsubsection{NUTRIÇÃO}

a) Aperfeiçoamento do manejo de forrageiras e desenvolvimento de novas variedades, de forma a otimizar o pastoreio dos ovinos (COIMBRA FILHO, 1985; CANTO et al., 1999; CARNEVALLI et al., 2001; GERDES, 2003; CUNHA et al., 2001).

b) Determinação de exigências nutricionais sob condições brasileiras, e adaptação de modelos para avaliação de dietas (PIRES et al., 2000; ALVES et al., 2003, CABRAL et al., 2008; RESENDE et al., 2008).

c) Estudos visando melhor aproveitamento de recursos alimentares e subprodutos regionais da agroindústria na nutrição ovina (NUNES et al., 1997; GARCIA, 1998; BROCHIER e CARVALHO, 2008; CORTE, 2007).

d) Desenvolvimento e utilização de estratégias de alimentação e suplementação (COIMBRA FILHO, 1985; CARVALHO et al., 1999; CUNHA et al., 2001; SUSIN, 2001; BÔAS et al., 2003; ALMEIDA JUNIOR et al., 2004). 
e) Estudos envolvendo o comportamento ingestivo e as preferências dos animais em relação à granulometria, forma de oferecimento e outras questões multidisciplinares (FISCHER et al., 1997; MACEDO et al., 2004; TURINO et al., 2004; CARDOSO et al., 2006; BOLZAN et al., 2007; MACEDO et al., 2007).

\subsubsection{REPRODUÇÃO}

a) Melhor compreensão da estacionalidade reprodutiva dos ovinos, e como se dá em diferentes raças e regiões do país (SASA et al., 2002; MARTINS et al., 2003; SOUZA et al., 2006; MOURA, 2009)

b) Aperfeiçoamento de protocolos hormonais para indução e sincronização de cio (NUNES et al., 1997; SOUSA e MORAES, 1998; DIAS et al., 2001; LOUREIRO, 2003).

c) Desenvolvimento e aplicação de biotecnologias reprodutivas (MORAES et al., 1998; SANTOS et al., 2006; TABET, 2007; GUSMÃO et al., 2009; MORAES et al., 2009; TRALDI, 2009)

\subsubsection{GENÉTICA}

a) Introdução de raças especializadas para produção de carne e estudos sobre a técnica do cruzamento industrial (OJEDA e OLIVEIRA, 1998; OJEDA, 1999; BONAGURIO, 2001; LÔBO, 2003; BARBOSA NETO, 2008; CARDOSO, 2008)

b) Início da conscientização sobre a importância de se conservar o patrimônio genético das raças e tipos nacionais (SOUSA, 1998; SOUSA, 1999; BUENO et al., 2000; EGITO, MARIANTE e ALBUQUERQUE, 2002)

c) Avaliação genética dos animais, com implantação de programas de melhoramento genético (OJEDA, 1997; OJEDA \& OLIVEIRA, 1998; MORAIS, 2000; ASCCO, 2007)

d) Pesquisas para melhor definição de objetivos de seleção e características a serem mensuradas (SOUSA, 1998; BARRETO NETO, 2004; ARAÚJO e SIMPLÍCIO, 2000). 
e) Implementação de testes de performance e técnicas inovadoras para mensuração das características (BUENO et al., 2007; JESUS, 2007; BRANQUINHO et al., 2008; ABCDORPER, 2010; ASPACO, 2010b)

\subsubsection{INSTALAÇÕES}

a) Definição das necessidades de instalações, dimensionamento e sua adaptação ao tipo de animal alojado, manejo, economicidade e condições climáticas específicas (PEREIRA, 1986; CUNHA et al., 2001; SOBRINHO, 2001; CUNHA et al. 2003; ALBANEZE et al, 2004).

b) Estudo de aspectos construtivos levam que respeitem o comportamento e o bem-estar dos animais e trabalhadores (PARANHOS DA COSTA e CROMBERG, 1997; RASLAN, 2008).

\subsubsection{MANEJO}

a) Estudos envolvendo diferentes sistemas e técnicas de manejo (RODA et al. 1993; SIQUEIRA et al., 1993; SOBRINHO, 2001; BONAGURIO, 2005).

b) Incorporação de princípios comportamentais e de bem-estar às técnicas de manejo; preocupação com boas práticas de criação (CUNHA et al., 1997, CÉSAR et al., 2008; RAINERI, 2008; RECH et al., 2008; MARQUES et al., 2009)

c) Melhoria das condições de embarque, transporte e desembarque dos animais (LEME, 2009)

\subsubsection{SANIDADE}

a) Desenvolvimento da indústria farmacêutica voltada para ovinos (BOFILL, 1996; ALVES e OLANDER, 1998)

b) Desenvolvimento de métodos alternativos de controle e prevenção de doenças e afecções (MACEDO, 2007; ZEOLA et al., 2007; NOGUEIRA et al., 2009).

C) Criação do Programa Nacional de Sanidade de Caprinos e Ovinos (BORGES, 2008). 


\subsubsection{CAPACITAÇÃO E ASSISTÊNCIA TÉCNICA}

a) Oferecimento de cursos de formação de mão de obra (OSORIO e ASTIZ, 1996; FURQUIM et al., 2005; SEBRAE, 2005; JESUS JUNIOR et al., 2010).

b) Promoção de palestras, dias de campo, cursos e treinamentos para estudantes, técnicos e produtores por diversas instituições de pesquisa, associações de criadores e outros agentes;

c) Disponibilidade (ainda que pequena) de profissionais especializados na criação de ovinos.

\subsection{INOVAÇÕES EM CORTES E PROCESSAMENTO}

a) Adequação dos cortes para a satisfação do consumidor brasileiro, bem como sua comercialização sob as formas congelada ou resfriada (SANTOS e PÉREZ, 2000; SANTOS, 2002; CARVALHO e PÉREZ, 2004; MORENO E SILVA SOBRINHO, 2008).

b) Industrialização da carne, com o desenvolvimento e produção de embutidos, defumados, pratos prontos e semi-prontos, como carne-seca, hambúrguer, cortes temperados, buchada e sarapatel (MONTEIRO e TERRA, 1999; FRANCESCHINI et al., 2006; De PELEGRINI et al., 2008; FRANÇOIS et al., 2009; SANTOS JUNIOR et al., 2009).

c) Início do processo de garantias de origem e qualidade, através da criação de marcas (VIEIRA FILHO, 2007; YOKOYA et al., 2009).

d) Desenvolvimento de tecnologias de embalagens compatíveis com os novos produtos e as exigências do consumidor, que ofereçam melhores apresentação e conservação, como embalagens a vácuo, com atmosfera modificada, com barreiras ao desenvolvimento de microrganismos e protetores de osso para evitar a perfuração. 


\subsection{INOVAÇÕES NA ÁREA DE GESTÃO}

\subsubsection{CONTROLE DOS CUSTOS DE PRODUÇÃO}

a) Conscientização sobre a necessidade de se controlarem custos de produção na ovinocultura e busca pelas metodologias mais adequadas (COSTA, 2007; BARROS, 2008; VIANA e SILVEIRA, 2008a, 2008b).

b) Projeto de elaboração de um índice de custos de produção para o estado de São Paulo, que possa servir como base para outros estados e situações (RAINERI e GAMEIRO, 2010).

\subsubsection{INFORMAÇÃO}

a) Iniciativas com o intuito de reduzir a assimetria de informações e aumentar a transparência no mercado de carne ovina, através do melhor fluxo de informações (ANTONELLI MENDES et al., 2009, 2010; GAMEIRO, 2009; UNICETEX, 2010)

b) Estudos descritivos do mercado, que contribuem para a informação dos envolvidos sobre o contexto da atividade (COSTA, 2007; CARRER et al., 2009; MEDEIROS e BRISOLA, 2009; SORIO, 2009, CARRER et al. 2010)

c) Surgimento de ferramentas específicas para a comunicação entre atores do SAG ovino (ou associado ao SAG caprino) na mídia, como portais na internet, revistas, jornais e programas televisivos;

d) Inserção da ovinocultura nos meios de comunicação não específicos, como os que tratam de agronegócios ou mesmo de notícias gerais;

e) Participação dos atores do SAG em cada vez mais eventos, como exposições de animais e feiras de produtos.

\subsection{IMPACTOS DAS INOVAÇÕES SOBRE O SISTEMA AGROINDUSTRIAL}

O desenvolvimento e aplicação das tecnologias descritas provocaram grande impacto sobre a estrutura e organização do SAG da carne ovina. Como demonstrado na Tabela 01, estes efeitos podem ser percebidos nas transações entre diferentes segmentos do SAG, e estão de acordo com as observações de Viana (2008). 
RAINERI, C. et al. As inovações tecnológicas na ovinocultura brasileira e seus efeitos na organização do sistema agroindustrial. PUBVET, Londrina, V. 7, N. 21, Ed. 244, Art. 1614, 2013.

Os maiores objetivos do SAG atualmente são alcançar qualidade (dos animais e produtos), padronização e disponibilidade. Para atingi-los, toda a cadeia vem sofrendo mudanças profundas, de forma que alterações na fase produtiva e nas transações tornam-se inter-relacionadas.

No tocante aos atributos das transações, tanto a especificidade dos ativos quanto a incerteza e freqüência sofrem incrementos. A especificidade dos ativos é, provavelmente, o atributo mais dramaticamente afetado. Segundo os pressupostos da ECT, Williamson (1996) considera a existência de seis tipos de especificidades de ativos: i) especificidade locacional, ii) especificidade de ativos físicos, iii) especificidade dedicada, iv) especificidades de ativos humanos, v) especificidade de marca e vi) especificidade temporal.

Tabela 01. Impacto das inovações tecnológicas nos aspectos da ECT no Sistema Agroindustrial da carne ovina.

\begin{tabular}{|c|c|c|c|c|c|}
\hline & \multicolumn{3}{|c|}{ Atributos das transações } & \multicolumn{2}{|c|}{ Pressupostos Comportamentais } \\
\hline & Especificidade & Frequência & Incerteza & $\begin{array}{c}\text { Racionalidade } \\
\text { limitada }\end{array}$ & Oportunismo \\
\hline \multicolumn{6}{|l|}{ Produção } \\
\hline Nutrição & Aumenta & Aumenta & Reduz & Presente & Presente \\
\hline Reprodução & Aumenta & Aumenta & Aumenta & Presente & Presente \\
\hline Genética & Aumenta & Aumenta & Aumenta & Presente & Presente \\
\hline Instalações & Aumenta & - & Aumenta & Presente & Presente \\
\hline Manejo & Aumenta & Aumenta & Reduz & Presente & Presente \\
\hline Sanidade & Aumenta & Aumenta & Reduz & Presente & Presente \\
\hline Capacitação & Aumenta & Aumenta & Reduz & Reduz & Reduz \\
\hline Processamento & Aumenta & Aumenta & Aumenta & Presente & Presente \\
\hline \multicolumn{6}{|l|}{ Gestão } \\
\hline Custos & Aumenta & Aumenta & Reduz & Reduz & Presente \\
\hline Informação & Aumenta & Aumenta & Reduz & Reduz & Reduz \\
\hline
\end{tabular}

Fonte: dados da pesquisa.

A especificidade locacional das transações é condicionada pela distância entre produtores, frigoríficos e centros consumidores, e por isto tende a ser 

2013.

mais baixa nas regiões Nordeste, Sudeste e Sul, onde há maior concentração de animais e uma indústria frigorífica melhor estruturada para processamento de carne ovina. Segundo Viana (2008), o estabelecimento de arranjos horizontais e verticais pode contribuir para reduzir esta especificidade, através da regularização da oferta e padronização dos animais. Exemplos de arranjos horizontais são a formação de núcleos e associações de produtores, cada vez mais freqüentes (SILVEIRA, 2005; ANPOVINOS, 2010; ASPACO, 2010).

Os animais passam a possuir uma especificidade maior que a demonstrada anteriormente. Os cordeiros para abate passam a precisar apresentar peso, idade, conformação de carcaça e quantidade adequadas à industrialização e à viabilidade econômica da propriedade criadora. Para isso, passaram a ser empregados reprodutores de raças especializadas, que exigem manejos melhorados, instalações adequadas, alimentação equilibrada, cuidados sanitários rígidos, e mão de obra capacitada. Desta forma, os ativos físicos sofrem elevação da especificidade.

Este cenário existe para suprir uma indústria frigorífica cujo objetivo é agregar valor ao produto, de forma a atingir mercados mais valorizados e mais exigentes. Assim, são necessários investimentos em plantas frigoríficas, equipamentos para processamento, embalagem, entre outros, o que eleva a especificidade dos ativos dedicados.

Para operar estes processos, sejam eles manejos dos animais, produção de insumos, abate e desossa ou gestão, são necessárias pessoas capacitadas. Assim, a especificidade de bens humanos também tende a se elevar.

Para agregar valor e certificar a qualidade dos produtos, diversas empresas vêm investindo também no fortalecimento de suas marcas (VIEIRA FILHO, 2007; YOKOYA et al., 2009), apesar de ainda não ser percebida grande diferenciação entre elas.

Por se tratar de um produto perecível, possui especificidade temporal no sentido em que os custos de transação aumentam à medida que aumentar o tempo decorrido até a comercialização. Segundo Viana (2008), esta especificidade é baixa, pois normalmente se passam apenas 48 horas entre 0 

2013.

embarque dos animais para o frigorífico e o momento da armazenagem de suas carcaças na câmara fria do abatedouro. No entanto, estes custos se elevam no caso de manutenção de estoques para comercialização posterior, como ocorre em algumas situações. O mesmo pesquisador destaca que quando passa a ser exigido algum tipo de ativo específico, deve haver uma contrapartida em termos de freqüência de transações para que o investimento feito seja mais rapidamente amortizado.

Os resultados deste estudo demonstram que a aplicação de tecnologias leva ao aumento da especificidade dos ativos, e normalmente um dos objetivos principais do processo é exatamente dispor de produtos (cordeiros, reprodutores, carne) para elevar a freqüência e o volume das transações. Já o estabelecimento observado de estruturas de governança como as parcerias verticais e horizontais estão de acordo com o apontado por Ferreira et al. (2005): segundo estes autores, a ECT aponta que, ao relacionar níveis de investimentos específicos com padrões de freqüência nas transações, pode-se estabelecer uma estrutura de governança que minimize os custos de transação.

A incerteza relaciona-se com a imprevisibilidade das atitudes posteriores dos agentes econômicos e com o desconhecimento de elementos relacionados ao ambiente econômico e institucional. No campo organizacional, a fonte fundamental de incerteza decorre exatamente da racionalidade limitada. Não fosse esse aspecto, as estruturas de governança seriam capazes de se ajustar às alterações havidas no ambiente. Há uma segunda fonte de incerteza que também se relaciona com aspectos comportamentais: o oportunismo. A aceitação do pressuposto de que os agentes podem se comportar de forma oportunista insere um componente de incerteza nas relações comerciais, na medida em que não há como prever exatamente o comportamento dos agentes mesmo após a realização de contratos (FERREIRA et al., 2005). Tecnologias que permitem maior independência dos agentes e/ou redução de custos proporcionam maior segurança. Alguns exemplos são a conservação de forragens, prevenção e controle de doenças e capacitação do produtor. Por 

2013.

outro lado, algumas novas práticas que objetivam principalmente a agregação de valores, podem aumentar custos ou a dependência em relação à indústria ou profissionais e processos muito especializados, como no caso das tecnologias reprodutivas e de processamento das carnes.

O conceito de racionalidade limitada constituiu junto com o oportunismo o alicerce da ECT. Os indivíduos agem racionalmente, porém de modo limitado. Assim, a obtenção de informações necessárias e a capacidade de processar problemas complexos são limitadas, ou seja, a racionalidade se torna escassa, implicando custos a sua utilização (VIANA, 2008).

Ao delinear a literatura da economia da informação, a ECT reconhece que muitas transações são caracterizadas por informações incompletas, imperfeitas ou assimétricas. A assimetria de informações pode levar a comportamentos oportunistas de duas maneiras: quando as informações não estão claras e transparentes antes da transação surge o oportunismo ex ante, e após uma transação que contenha assimetria de informações, ações de indivíduos ou firmas podem levar ao oportunismo ex post, comportamento esse caracterizado por "risco moral" (HOBBS, 1996).

A aplicação das tecnologias, principalmente as de informação, tem potencial para contribuir para a redução da incerteza, da racionalidade limitada e do oportunismo nas transações, através do fornecimento de subsídios para as tomadas de decisão. No entanto, de acordo com a teoria da ECT, estes fatores não poderiam ser eliminados. No momento, o desenvolvimento das tecnologias da informação no SAG da carne ovina ainda não é suficiente, na grande maioria dos casos, para reduzir tais aspectos das transações.

Outros efeitos das inovações podem ser observados no SAG: i) o aumento de sua complexidade, com novos participantes, produtos e serviços, desenvolvidos e disponibilizados para suprir novas demandas; e ii) o surgimento de novas formas de governança, apesar do principal mecanismo de preços ainda ser o mercado. Há cada vez mais casos de coordenações verticais e horizontais: apesar de não ser usual a utilização de contratos entre as partes, a incerteza, o oportunismo e a racionalidade limitada podem ser 

2013.

reduzidos em situações assim. A ECT aponta que a forma de governança ideal é definida pelos atributos, como forma de minimizar os custos de transação. Assim, mudanças nos atributos podem alterar a forma de governança.

Quando aplicados no campo, os novos conhecimentos passam a gerar uma demanda por produtos e serviços antes inexistentes. Eles tornaram-se necessários para atingir melhores níveis produtivos, e foram desenvolvidos e disponibilizados. Na última década passou a ser possível encontrar suplementos minerais e vitamínicos específicos para ovinos; medicamentos; equipamentos, como cochos de vários materiais e modelos; materiais para construção de cercas, como telas, eletrificadores, entre outros; balanças, currais, baias móveis, troncos; softwares, brincos, colares e microchips para controle dos rebanhos e inúmeros equipamentos para manejos. Entre os serviços, podemos citar os de consultoria, comercialização, preparação de animais para exposições, transporte, registro genealógico, tosquia, avaliação de carcaça, reprodução assistida, entre muitos outros.

\section{CONCLUSÕES}

O comportamento da ovinocultura brasileira está intimamente relacionado à evolução do conhecimento e informação. Ao longo da história, a tecnologia (ou sua falta) foi decisiva em diversos momentos para alavancar ou impedir o progresso da atividade.

Quando aplicadas, novas tecnologias passam a gerar novas situações e demandas. Assim, nos últimos anos o desenvolvimento científico e tecnológico foi capaz de atuar como condicionante da estruturação e organização do sistema agroindustrial da carne ovina, o que pode ser demonstrado pela Teoria da Economia dos Custos de Transação através de sua influência sobre os atributos das transações.

A ovinocultura vive um momento de grande desenvolvimento tecnológico em seus diversos campos, porém ainda há muito a ser feito especialmente a respeito da geração e aplicação de conhecimentos na área da Gestão. 


\section{REFERÊNCIAS BIBLIOGRÁFICAS}

ABCDORPER - ASSOCIAÇÃO BRASILEIRA DOS CRIADORES DE DORPER E WHITE DORPER. 2010. Regulamento da $1^{a}$ prova de ganho de peso do circuito brasileiro das raças Dorper e White Dorper. [on line] Disponível em: http://www.farmpoint.com.br/?noticiaid=62555\&acta=7\&areaid=1\&secaoid=13. acesso em 18/07/2010.

ALBANEZE, R.F.G.N.; SILVA, J.C.; SANTOS, S.A.; SILVA, R.A.M.S.; LEITE, L.A.R. Um modelo de instalação para a criação de ovinos em semi-confinamento na Parte Alta de Corumbá, MS. IN: SIMPÓSIO SOBRE RECURSOS NATURAIS E SÓCIO-ECONÔMICOS DO PANTANAL, 4, 2004, Corumbá. Anais... Corumbá: 2004.

ALMEIDA JUNIOR, G.A.; COSTA, C.; MONTEIRO, A.L.G.; GARCIA, C.A.; MUNARI, D.P.; NERES, M.A. Desempenho, características de carcaça, e resultado econômico de cordeiros criados em creep feeding com silagem de grãos úmidos de milho. Revista Brasileira de Zootecnia. 2004, V.33, n. 4, pp. 1039-1047.

ALVES, F.S.F., OLANDER, H.J. Uso de uma vacina toxoide no controle da linfadenite caseosa em caprinos. Revista Brasileira de Medicina Veterinaria. 1998, v.20, n.2, p.74-77.

ALVES, K.S. et al. Níveis de energia em dietas para ovinos Santa Inês: desempenho. Revista Brasileira de Zootecnia. [online]. 2003, vol.32, n.6, supl.2, pp. 1937-1944.

ANPOVINOS - ASSOCIAÇÃO NORDESTE PAULISTA DE OVINOCULTORES. Quem Somos. [on line] Disponível em: <http://www.anpovinos.com.br/QuemSomos.asp> Acesso em 16/07/2010.

ANTONELLI MENDES, R., CARRER, C.C., GAMEIRO, A.H. Um panorama da ovinocultura brasileira e o Índice de Preço do Cordeiro Paulista. Boletim de Socioeconomia e Ciência Animal, n.7, de 31 de agosto de 2009. Disponível em: http://www.fmvz.usp.br/index.php/site/content/download/6429/27755/file/Socioeconomia_\& _Ciencia_Animal_Edicao_007.pdf

ANTONELLI MENDES, R.A.; GAMEIRO, A.H.; FIRETTI, R.; RAINERI, C.; CARRER, C.C. Índide de Preços do Cordeiro: um serviço de apoio à ovinocultura de corte do estado de São Paulo. IN: REUNIÃO ANUAL DA SOCIEDADE BRASILEIRA DE ZOOTECNIA, 47, 2010. Salvador. Anais... Salvador, 2010.

ARAÚJO, A. M. e SIMPLÍCIO, A.A. Melhoramento genético em caprinos e ovinos no Brasil: importância do padrão racial. IN: Simpósio Nacional de Melhoramento Animal, 3, 2000. Belo Horizonte. Anais... Belo Horizonte, 2000.

ASCCO - ASSOCIAÇÃO SERGIPANA DOS CRIADORES DE CAPRINOS E OVINOS. Sumário de carneiros e matrizes da raça Santa Inês - verão/2008. Pirassununga:GMA-FZEA-USP, 2007. $92 \mathrm{f}$.

ASPACO - ASSOCIAÇÃO PAULISTA DOS CRIADORES DE OVINOS. Núcleos regionais. [on line] Disponível em: <http://www.aspaco.com.br/nucleos.php> Acesso em 16/07/2010.

ASPACO - ASSOCIAÇÃO PAULISTA DOS CRIADORES DE OVINOS. Regulamento do IX Campeonato Cordeiro Paulista. [On Line] Disponível em: http://aspaco.org.br/circulares.php?id=883. Acesso em 18/07/2010b.

BARBOSA NETO, A.C. Avaliação de cruzamentos de ovinos das raças Dorper, Poll Dorset, Santa Inês e Somalis Brasileira. Dissertação (Mestrado) - Universidade Federal do Ceará, Centro de Ciências Agrárias, Fortaleza, 2008. 
BARRETO NETO, A.D. Análise sistêmica e mercadológica aplicada a definição de objetivos de seleção em ovinos Santa Inês. IN: Simpósio da Sociedade Brasileira de Melhoramento Animal, 5, 2004. Pirassununga, Anais... Pirassununga, 2004.

BARROS, C.S. Análise econômica de sistemas de produção de ovinos para carne. Dissertação (Mestrado em Ciências Veterinárias) - Setor de Ciências Agrárias, Universidade Federal do Paraná. Curitiba, 2008. 145 p.

BÔAS, A.S.V.; ARRIGONI, M.D.B.; SILVEIRA, A.C.; COSTA, C.; CHARDULO, L.A.L. Idade à Desmama e Manejo Alimentar na Produção de Cordeiros Superprecoces. Revista Brasileira de Zootecnia. 2003, v.32, n.6, pp.1969-1980, (Supl. 2).

BOFILL, F. J. A reestruturação da ovinocultura gaúcha. Guaíba: Livraria e Editora Agropecuária, 1996. $137 \mathrm{p}$.

BOLZAN, I.T.; SANCHEZ, L.M.B.; CARVALHO, P.A.; VELHO, J.P.; LIMA, L.D.; MORAIS, J.; CADORIN JUNIOR, R.L. Consumo e digestibilidade em ovinos alimentados com dietas contendo grão de milho moído, inteiro ou tratado com uréia, com três níveis de concentrado. Ciência Rural. 2007, vol.37, no.1, pp. 229-234.

BONAGURIO, S. Estudo da suplementação de ovelhas, idade à desmama e sistemas de terminação de cordeiros machos inteiros sobre a qualidade de carne. Botucatu, 2005. Dissertação (Doutorado) - Universidade Estadual Paulista, Faculdade de Medicina Veterinária e Zootecnia.

BONAGURIO, S. Qualidade da carne de cordeiros Santa Inês puros e mestiços com Texel abatidos com diferentes pesos. Lavras, 2001. 150 p. Dissertação (Mestrado) - Universidade Estadual de Lavras, Faculdade de Zootecnia.

BORGES, C.H.P. Programa Nacional de Sanidade dos Caprinos e Ovinos - PNSCO. IN: CONGRESSO BRASILEIRO DE ZOOTECNIA, 8, 2008, João Pessoa. Anais... João Pessoa, 2008.

BRANQUINHO, R.P. ; PAIM, T.P.; LOUVANDINI, H.; DALLAGO, B.S.; PAIVA, S.R.; MCMANUS, C.M. Interação genótipo ambiente em provas de ganho em peso confinados e a pasto em ovinos no DF. In: CONGRESSO BRASILEIRO DE MEDICINA VETERINÁRIA, 35, 2008. Gramado. Anais... Gramado, 2008.

BROCHIER, M.A.; CARVALHO, S. Consumo, ganho de peso e análise econômica da terminação de cordeiros em confinamento com dietas contendo diferentes proporções de resíduo úmido de cervejaria. Arquivo Brasileiro de Medicina Veterinária e Zootecnia. 2008, v.60, n.5, p.1205-1212.

BUENO, M.S.; CUNHA, E.A.; SANTOS, L.E. Santa Inês sheep breed in the intensive meat production in the southeast region of Brazil. In: GLOBAL CONFERENCE ON CONSERVATION OF DOMESTIC ANIMAL RESOURCES, 5., 2000, Brasília. Proceedings... Brasília: EMBRAPA, 2000. CD-ROM.

BUENO, M. S.; CUNHA, E. A.; SANTOS, L. E. dos; VERÍSSIMO, C. J.. Utilização da ultrasonografia na avaliação de características de carcaça de ovinos. 2007. Artigo em Hypertexto. Disponível em: <http://www.infobibos.com/Artigos/2007_2/ultra/index.htm>. Acesso em: $18 / 7 / 2010$

CABRAL, L.S.; NEVES, E.M.O.; ZERVOUDAKIS,J.T.; ABREU,J.T.; RODRIGUES, R.C.; SOUZA, A.L.; OLIVEIRA, I.S. Estimativas dos requisitos nutricionais de ovinos em condições brasileiras. Revista Brasileira de Saúde e Produção Animal. 2008, vol. 9, n. 3.

CANTO, M.W.; MOOJEN, E.L.; CARVALHO, C.F.; SILVA, J.H.S. Produção de cordeiros em pastagem de azevém e trevo-branco sob diferentes níveis de resíduos de forragem. Pesquisa Agropecuária Brasileira. [online]. 1999, vol.34, n.2, pp. 309-316. 
CARDOSO, M.T.M. Desempenho e características de carcaça de ovinos da raça Santa Inês e seus cruzamentos em sistema intensivo de produção. 2008, 117 f. Dissertação (Mestrado) Universidade de Brasília, Faculdade de Agronomia e Medicina Veterinária.

CARDOSO, A.R.; CARVALHO, S.; GALVANI, P.B. et al. Comportamento ingestivo de cordeiros alimentados com dietas contendo diferentes níveis de fibra em detergente neutro. Ciência Rural. 2006, v.36, n.2, pp.604-609.

CARNEVALLI, R.A., SILVA, S.C., FAGUNDES, J.L., SBRISSIA, A.F., CARVALHO, C.A.B, PINTO, L.F.M., PEDREIRA, C.G.S. Desempenho de ovinos e respostas de pastagens de Tifton 85 (Cynodon spp.) sob lotação contínua. Scientia Agricola. 2001, vol.58, n.1, pp. 7-15.

CARRER, C.C. A cadeia de negócios da ovnivocultura de corte paulista: diagnóstico de pontos críticos e proposta de estruturação técnica e mercadológica. Pirassununga: Ed. Lawbook, $1^{a}$ Ed, 2009. 183 p.

CARRER, C.C. ANTONELLI MENDES, R.; RAINERI, C.PROSDOCIMI NUNES, B.C.; GAMEIRO, A.H. Caracterização da carne ovina produzida e da dinâmica de seu mercado em estados nordestinos. In: CONGRESSO BRASILEIRO DE ZOOTECNIA, 20., 2010, Palmas. Anais... Palmas: Associação Brasileira de Zootecnistas, 2010.

CARVALHO, P. A.; PÉREZ, J. R. O. Cortes comerciais em carcaças ovinas. Boletim Técnico, 96, Lavras, Universidade Federal de Lavras (UFLA), 2004. Disponível em:<http:// http://www.editora.ufla.br/BolExtensao/pdfBE/bol_96.pdf >. Acesso em: 26 ago. 2010.

CARVALHO, S.; PIRES, C.C.; PERES, J.R.R. et al. Desempenho de cordeiros machos inteiros, machos castrados e fêmeas, alimentados em confinamento. Ciência Rural. 1999, vol. 29, n.1, pp.129-133.

CÉSAR, A.P.; RIGO, A.; RAINERI, C.; NADALETTO, C.E.S.; TERENCIO, D.A.B.; SOUZA, F.M.; MARQUES, L.J.; CONDELI, M.; BALDIN, S.R. Manual de boas práticas para ovinos de corte. 1a. Ed. SEBRAE/ ANPOVINOS. São José do Rio Preto: SR Gráfica e Editora. 2008. 62 p.

COIMBRA FILHO, A. Técnicas de criação de ovinos. Porto Alegre: EMATER/RS, 1985. 92 p.

CORTE, R.R.P.S. Efeito do caroço de algodão no desempenho e características de carcaça e da carne de cordeiros cruzados. 79 p. Dissertação (Mestrado) - Faculdade de Zootecnia e Engenharia de Alimentos, Universidade de São Paulo, Pirassununga, 2007.

COSTA, A. D. Nível tecnológico, rentabilidade e cadeia produtiva da ovinocaprinocultura de corte no estado do Ceará. Dissertação (Mestre em Economia Rural) - Universidade Federal do Ceará. Fortaleza, 2007. 81 p.

CUNHA, E.A.; BUENO, M.S.; SANTOS, L.E. et al. Sistema intensivo de produção ovina. Nova Odessa: Instituto de Zootecnia, 2001. $51 \mathrm{p}$.

CUNHA, E.A.; BUENO, M.S.; SANTOS, L.E.; VERÍSSIMO, C.J. Produção Ovina. Nova Odessa: Instituto de Zootecnia, 2003. 80 p.

CUNHA, E.A.; SANTOS, L.E.; RODA, D.S. et al. Efeito do sistema de manejo sobre o comportamento em pastejo, desempenho ponderal, e infestação parasitária em ovinos Suffolk. Pesquisa Veterinária Brasileira. 1997, v.17, n.3-4, p. 105-111.

De PELEGRINI, L.F.V.; PIRES, C.C.; TERRA, N.N.; CAMPAGNOL, P.C.B.; GALVANI, D.B.; CHEQUIM, R.M. Elaboração de embutido fermentado tipo salame utilizando carne de ovelhas de descarte. Ciência e Tecnologia de Alimentos. 2008, vol. 28, (Supl.), pp. 150-15.

DIAS, F.E.F.;, LOPES JUNIOR, E.S., VILLAROEL, A.B.S; RONDINA, D.; LIMA-VERDE, J.B.; PAULA, N.R.O.; FREITAS, V.J.F. Sincronização do estro, indução da ovulação e fertilidade de ovelhas deslanadas após tratamento hormonal com gonadotrofina coriônica eqüina. Arquivo Brasileiro de Medicina Veterinária e Zootecnia [online]. 2001, vol.53, n.5, pp. 618-623. 
EgITO, A.A., MARIANTE, A.S. E ALBUQUERQUE, M.S.M.. Programa brasileiro de conservação de recursos genéticos animais. Archivos de Zootecnia. 2002, vol.51, pp. 39-52.

FERREIRA, G.M.V. et al. Economia dos custos de transação sob uma análise crítica: perspectivas de aplicação no agronegócio. IN: CONGRESSO BRASILEIRO DE ECONOMIA E SOCIOLOGIA RURAL, 43., 2005. Ribeirão Preto: Anais... Ribeirão Preto: SOBER, 2005.

FISCHER, V.; DESWYSEN,A.G.; DĖSPRES, L. et al. Comportamento ingestivo de ovinos recebendo dieta a base de feno durante um período de seis meses. Revista Brasileira de Zootecnia. 1997, v.26, n.5, pp.1032-1038.

FRANCESCHINI, R.; BONACINA, M.; TREPTOW, R.; MONTEIRO, E.; QUEIROZ, M.I. Caracterização sensorial de salsicha ovina. Alimentos e Nutrição. 2006, v.17, n.2, p.127-135.

FRANÇOIS, P.; PIRES, C.C.; GRIEBLER, L.; FRANÇOIS, T.; SORIANO, V.S.; GALVANI, D.B. Propriedades físico-químicas e sensoriais de embutidos fermentados formulados com diferentes proporções de carne suína e de ovelhas de descarte. Ciência Rural. 2009, v.39, n.9, pp.2584-2589.

FURQUIM, G.; MEDEIROS, M.I.M.; PEROSA, J.M.Y.; STEIN, A.C. Padronização e qualidade da carne na ovinocultura: a capacitação como mecanismo de competitividade frente às demandas de mercado. IN: CONGRESSO DA SOBER, XLIII, 2005, Ribeirão Preto. Anais... Ribeirão Preto, 2005.

GARCIA, C.A. Avaliação do resíduo de panificação "biscoito" na alimentação de ovinos e nas características quantitativas e qualitativas da carcaça. Jaboticabal, 1998. 79 p. Dissertação (Mestrado em Zootecnia) - Faculdade de Ciências Agrárias e Veterinárias, Universidade Estadual Paulista.

GAMEIRO, A.H. Monitoramento de preços do mercado e o Índice do Cordeiro. Palestra proferida no Simpósio Cadeia de Ovinocultura de Carne, no dia 20 de maio de 2009, como parte da programação do ZOOTEC 2009.

GERDES, L. Introdução de uma mistura de três espécies forrageiras de inverno em pastagem irrigada de capim-aruana. Tese (Doutorado) - Escola Superior de Agricultura Luiz de Queirós. Universidade de São Paulo. 2003. Disponível em: http://www.teses.usp.br/teses/disponiveis/11/11139/tde-05012004-153828/

GUSMÃO, A.L.; SILVA, J.C.; BITTENCOURT, T.C.C.; MARTINS, L.E.P.; GORDIANO, H.D.; BARBOSA, L.P. Coleta transcervical de embriões em ovinos da raça Dorper no semiárido do nordeste brasileiro. Arquivo Brasileiro de Medicina Veterinária e Zootecnia. 2009, vol.61, n.2, pp. 313-318.

HOBBS, J.E. A transaction cost approach to supply chain management. Supply Chain Management, v. 1, n. 2, p. $15-27,1996$.

INSTITUTO BRASILEIRO DE GEOGRAFIA E ESTATÍSTICA - IBGE. Pesquisa Pecuária Municipal de 2009. [on line] Disponível em: http://www.sidra.ibge.gov.br/bda/tabela/protabl.asp?c $=73 \& z=t \& o=24 \& i=P$. Acesso em 08/06/2011.

JESUS, T.I. Considerações sobre carcaças ovinas e utilização da ultra-sonografia como ferramenta na avaliação de características de carcaças de ovinos da raça santa inês mantidos em confinamento para terminação. Monografia (Conclusão de curso) - Universidade Metodista de São Paulo, São Bernardo do Campo, 2007.

JESUS JUNIOR, C.; RODRIGUES, L.S.; MORAES, V.E.G. Ovinocaprinocultura de corte - a convivência dos extremos. BNDES Setorial. 2010, vol. 31, pp. 281-320.

LEME, T.M.C. Métodos de transporte e períodos de descanso pré-abate sobre nível de estresse e qualidade de carne de ovinos. 95 f. Dissertação (Mestrado) - Universidade de São Paulo, Faculdade de Zootecnia e Engenharia de Alimentos, Pirassununga, 2009. 
LÔBO, R. N. B. Cruzamento industrial: quando e como fazer? Seminário Nordestino de Pecuária, 7; Feira de Produtos e de Serviços Agropecuários, 7, 2003. Palestras técnicas. Fortaleza: Federação da Agricultura e Pecuária do Estado do Ceará, 2003, v. 5.

LOUREIRO, M.F.P. Indução do estro por implante de melatonina em ovinos da raça Suffolk. Dissertação (Mestrado) - Universidade de São Paulo, Faculdade de Medicina Veterinári e Zootecnia, Pirassununga. 2003.

MACEDO, F.R. Efeitos da administração da folha de Nim indiano (Azadirachta indica A. Juss) no controle de helmintos em ovinos infectados naturalmente. 45 p. Dissertação (Mestrado) Universidade de Brasília, Faculdade de Agronomia e Medicina Veterinária, Brasília, 2007.

MACEDO, C.A.B.; MIZUBUTI, I.Y.; MOREIRA, F.B.; PEREIRA, E.S.; RIBEIRO, E.L.A; ROCHA, M.A.; RAMOS, B.M.O.; MORI, R.M.; PINTO, A.P.; ALVES, T.C.; CASIMIRO, T.R. Comportamento ingestivo de ovinos recebendo dietas com diferentes níveis de bagaço de laranja em substituição à silagem de sorgo na ração. Revista Brasileira de Zootecnia. 2007, vol.36, n.6, pp. 1910-1916.

MACEDO JR., G.L.; PÉREZ, J.R.O.; PAULA, O.J. et al. Influência dos diferentes níveis de FDN proveniente de forragem no comportamento ingestivo de ovelhas Santa Inês não gestantes. In: REUNIÃO ANUAL DA SOCIEDADE BRASILEIRA DE ZOOTECNIA, 41., 2004, Campo Grande. Anais... Campo Grande: Sociedade Brasileira de Zootecnia, 2004. (CD-ROM).

MARQUES, L. J. ; TERENCIO, D. A. B. ; CONDELI, M. ; RIGO, A. ; RAINERI, C. ; SOUZA, F. M. ; NADALETTO, C. E. S. ; BALDIN, S. R. ; JACOMETI, W. A. ; CESAR, A. P. ; VERISSIMO, C. J. ; NUNES, B.C.P. ; TRALDI, A. S. ; OLIVEIRA, M. A. G. ; LINS, G. P. V. ; LINS, G. J. V. ; FREITAS, D. ; GIANLOURENCO, V. K. ; VIEIRA, S. C. ; BOVO, T.B. . Manual de Boas Práticas para Ovinos de Corte (2a Edição). SEBRAE/ ANPOVINOS. São José do Rio Preto: SR Gráfica e Editora. 2009.

MARTINS, R.D., MCMANUS, C.; CARVALHÊDO, A.S.; BORGES, H.V.; SILVA, A.E.; DIAS, F.; SANTOS, N.R. Avaliação da sazonalidade reprodutiva de carneiros Santa Inês criados no Distrito Federal. Revista Brasileira de Zootecnia [online]. 2003, vol.32, n.6, suppl.1, pp. 1594-1603.

MEDEIROS, J.X., BRISOLA, M.V. Gestão e organização no agronegócio da ovinocaprinocultura. Contagem: Santa Clara Editora, 2009. 219 p.

MONTEIRO, E.M.; TERRA, N.N Processamento do presunto "cook-in" de cordeiros. Ciência Rural. 2009, v. 29, n. 4, pp. 721-725.

MORAES, J.C.F., SOUZA, C.J.H., COLLARES, R.S. Situação atual e perspectivas da inseminação artificial em ovinos. Revista Brasileira de Reprodução Animal. 1998, v.22, n.2, pp.87-91.

MORAES, E.P.B.X., FREITAS NETO, L.M., AGUIAR FILHO, C.R., ALMEIDA-IRMÃO, J.M., SANTOS, M.H.B., NEVES, J.P., LIMA, , P.F., OLIVEIRA, M.A.L. Eficiência no diagnóstico de prenhez em ovinos pela ultrassonografia transretal e transvaginal. Medicina Veterinária. 2009, v.3, n.2, pp.15-20.

MORAIS, O.R. O melhoramento genético dos ovinos no Brasil: situação atual e perspectivas para o futuro. IN: Simpósio Nacional de Melhoramento Animal, 3, 2000. Belo Horizonte. Anais... Belo Horizonte, 2000.

MORAND-FEHR, P. Recent developments in goat nutrition and application: A review. Small Ruminant Research. 2005, v.60, I. 1-2, pp.25-43.

MORENO, G.; SILVA SOBRINHO, A. Produção de carnes ovina e caprina e cortes da carcaça. In: SEMINÁRIO NORDESTINO DE PECUÁRIA, 12., 2008, Fortaleza. Anais... Fortaleza: Federação da Agricultura e Pecuária do Estado do Ceará, 2008. 
MOURA, A.C.B. Desempenho reprodutivo de ovelhas Santa Inês criadas no nordeste paraense. Dissertação (Mestrado) - Universidade Federal do Ceará, Centro de Ciências Agrárias, Fortaleza, 2009.

NOGUEIRA, D.M.; NASCIMENTO, T.V.C.; MACEDO, J.L.; MACEDO, L.V.L. Uso do método Famacha para controle de nematódeos gastrintestinais em ovinos no alto sertão de Pernambuco: resultados parciais. IN: CONGRESSO BRASILEIRO DE ZOOTECNIA, 19, 2009, Águas de Lindóia. Anais... Águas de Lindóia, 2009.

NUNES, J.F., CIRÍACO, A.L.T., SUASSUNA, U. Produção e reprodução de caprinos e ovinos. $2^{a}$ Ed. Fortaleza:Editora Gráfica LCR, 1997. 199 p.

OJEDA, D.B. 1977 - 1997 Vinte anos de avaliações genéticas de ovinos lanados realizadas no Brasil. In: REUNIÃO DO SGT 8 DO MELHORAMENTO ANIMAL DO MERCOSUL, Buenos Aires, 1997.

OJEDA, D.B. Participação do melhoramento genético na produção ovina. Revista Brasileira de Reprodução Animal. 1999, v.23, n.2, pp.146-149.

OJEDA, D.B. \& OLIVEIRA, N.M. Serviço de Avaliação Genética de Reprodutores ovinos. S.A.G.R.O.: Resultados de 1998. Bagé, Embrapa Pecuária Sul, 31p.,1998.

OSORIO, J.C., ASTIZ, C.S. Qualidade da carcaça e carne ovina. In: PROGRAMA DE TREINAMENTO EM OVINOCULTURA. SENAR, Porto Alegre, RS. p. 110-190, 1996

PARANHOS DA COSTA, M.J.R. E CROMBERG, V.U. (1997). Alguns aspectos a serem considerados para melhorar o bem-estar de animais em sistema de pastejo rotacionado. In: Peixoto, A.M., Moura, J.C. e Faria, V.C. Fundamentos do Pastejo Rotacionado, FEALQ: Piracicaba, p. 273-296.

PEREIRA, M.F. Construções Rurais. 330 p. São Paulo: Nobel, 1986.

PIRES, C.C., SILVA, L.F., SANCHEZ, L.M.B. Composição Corporal e Exigências Nutricionais de Energia e Proteína para Cordeiros em Crescimento. Revista Brasileira de Zootecnia. 200, v.29, n.3, pp. 853-860.

RAINERI, C. Perfil do comportamento materno-filial de ovinos da raça Santa Inês e sua influência no desempenho dos cordeiros ao desmame. 2008. 61 f. Dissertação (Mestrado) Faculdade de Zootecnia e Engenharia de Alimentos, Universidade de São Paulo, Pirassununga, 2008.

RAINERI, C.; GAMEIRO, A.H. Indicadores econômicos para a ovinocultura: o índice de custo de produção do cordeiro paulista. Revista Cabra e Ovelha, ano V, no. 51, junho de 2010.

RASLAN, L.S.A. Aspectos comportamentais e fisiológicos de ovino srd sob pastejo com e sem sombreamento. Dissertação (Mestrado) - Universidade Estadual do Sudoeste da Bahia, 2008.

RECH, C.L.S.; RECH, J.L.; FISCHER, V.; OSÓRIO, M.T.M.; MANZONI, N.; MOREIRA, H.L.M.; SILVEIRA, I.D.B.; TAROUCO, A.K. Temperamento e comportamento materno-filial de ovinos das raças Corriedale e Ideal e sua relação com a sobrevivência dos cordeiros. Ciencia Rural. 2008, vol.38, no.5, pp. 1388-1393.

RESENDE, K.T.; SILVA, H.G.O.; LIMA, L.D.; TEIXEIRA, I.A.M.A.T. Avaliação das exigências nutricionais de pequenos ruminantes pelos sistemas de alimentação recentemente publicados. Revista Brasileira de Zootecnia. 2008, vol.37 (supl. especial), pp.161-177.

RESENDE, K.T.; TEIXEIRA, I.A.M.A.; BIAGIOLI, B.; LIMA, L.D.; NETO, O.B.; PEREIRA JUNIOR, J.D. Progresso científico em pequenos ruminantes na primeira década do século XXI. Revista Brasileira de Zootecnia. 2010, v.39 (supl. especial) pp. 369-375.

RODA, D.S.; SANTOS, L.E.; CUNHA, E.A. et al. Desempenho de ovinos em sistemas de acasalamento a cada oito meses. Boletim de Industria Animal. 1993, n. 50, v.1, pp. 49-54. 
SANTOS, M.H.B.; MORAES, E.P.B.X.; GUIDO, S.I.; BEZERRA, F.Q.G.; MELO, A.N.; LIMA, P.F.; OLIVEIRA, M.A.L. Sexagem fetal em ovelhas Santa Inês por ultra-sonografia. Sexagem fetal em ovelhas. Ciência Rural. 2006, v.36, n.2, pp.573-578.

SASA, A., TESTON, D.C., RODRIGUES, P.A., COELHO, L.A.; SCHALCH, E. Concentrações Plasmáticas de Progesterona em Borregas Lanadas e Deslanadas no Período de Abril a Novembro, no Estado de São Paulo. Revista Brasileira de Zootecnia. 2002, v.31, n.3, pp.1150-1156.

SERVIÇO BRASILEIRO DE APOIO ÀS MICRO E PEQUENAS EMPRESAS - SEBRAE. Informações de mercado sobre caprinos e ovinos, relatório completo. Unidade de Acesso a Mercados UAM, Brasília: 2005. 73p.

SILVEIRA, H.S. Coordenação na cadeia produtiva de ovinocultura: o caso do conselho regulador Herval Premium. 2005. 104 f. Dissertação (Mestrado em Agronegócios) Universidade Federal do Rio Grande do Sul, Porto Alegre, 2005.

SIQUEIRA, E.R.; AMARANTE, A.F.T.; FERNANDES, S. Estudo comparativo da recria de cordeiros em confinamento e em pastagem. Revista Brasileira de Zootecnia. 1993, v.5, p.1728.

SOBRINHO, A.G.S. Criação de Ovinos. 2a Edição. Jaboticabal: FUNEP, 2001. 302 p.

SORIO, A. Sistema agroindustrial da carne ovina em Mato Grosso do Sul. Uma abordagem da nova economia institucional. Dissertação (Mestrado) - Universidade Federal de Mato Grosso do Sul, Departamento de Economia e Administração. Campo Grande: 2009, 120 p.

SANTOS, C. L. dos. Sistemas de cortes de cordeiros. In: SIMPÓSIO MINEIRO DE OVINOCULTURA, 2., 2002, Lavras, MG. Anais... Lavras:UFLA, 2002. pp. 187-206.

SANTOS, C. L.; PÉREZ, J. R. O. Cortes comerciais de cordeiros Santa Inês. In: ENCONTRO MINEIRO DE OVINOCULTURA, 1., 2000, Lavras, MG. Anais... Lavras: UFLA, 2000. p. 149168.

SANTOS JÚNIOR, L.C.O.; RIZZATTI, R.; BRUNGERA, A.; SCHIAVINI, T.J.; CAMPOS, E.F.M.; SCALCO NETO, J.F.; RODRIGUES, L.B.; DICKEL, E.L.; SANTOS, L.R. Desenvolvimento de hambúrguer de carne de ovinos de descarte enriquecido com farinha de aveia. Ciência Animal Brasileira. 2009, v.10, n. 4, pp. 1128-1134.

SOUSA, W.H. Conservação ativa dos recursos genéticos de caprinos e ovinos no Brasil. In: CONGRESSO PERNAMBUCANO DE MEDICINA VETERINÁRIA, 4, 1999, Recife, PE. Anais... Recife: Sociedade Pernambucana de Medicina Veterinária, 1999. p.105-107.

SOUSA, W.H. Ovinos Santa Inês: potencialidades e limitações. In: Simpósio Nacional de Melhoramento Animal, 20, 1998, Uberaba. Anais... Viçosa: Sociedade Brasileira de Melhoramento Animal, 1998, p.233-237

SOUSA, C.J.H., MORAES, J.C.F. Manual de sincronização de cios em ovinos e bovinos. Bagé: Embrapa Pecuária Sul, 1998. 76 p. (Documentos)

SOUZA, M. I. L; URIBE-VELÁSQUEZ, L. F; BICUDO, S. D; RAMOS, A. A. Determinação dos ritmos circadianos e circanuais nas concentrações plasmáticas de testosterona e androstenediona em machos ovinos. Arquivos de Ciências Veterinárias e Zoologia. 2006, v. 9, n. 2, pp. 123-127.

SUSIN, I. Confinamento de cordeiros. In; Mattos, W.R.S. el al. (Ed.) A produção animal na visão dos brasileiros. Piracicaba: FEALQ, 2001, p. 454-460.

TABET, A.F. Transfêrencia intratubárica videolaparoscópica de embriões ovinos fertilizados in vitro. Tese (doutorado) - Universidade de São Paulo, Faculdade de Medicina Veterinária e Zootecnia, São Paulo, 2007. 
TRALDI, Anneliese de Souza. Produção in vitro de embriões de ovinos:uma visão crítica do método e de seu resultado a campo. Revista Brasileira de Zootecnia [online]. 2009, vol.38, n.spe, pp. 301-306.

TURINO, V.F.; SUSIN, I.; PIRES, A.V.; MENDES, C.Q. et al. Comportamento ingestivo de cordeiros alimentados com dietas à base de concentrado tendo como fonte de fibra o bagaço de cana-de-açúcar ou a casca de soja. In: REUNIÃO ANUAL DA SOCIEDADE BRASILEIRA DE ZOOTECNIA, 41., 2004, Campo Grande. Anais... Campo Grande: Sociedade Brasileira de Zootecnia, 2004. (CD-ROM).

UNICETEX - CENTRO DE INOVAÇÃO TECNOLÓGICA E EXTENSÃO UNIVERSITÁRIA DA FZEA/USP. Boletim mensal do Indicador de Preços do Cordeiro Paulista. Ed. 11, abril de 2010.

VIEIRA FILHO, A.S. Cadeia produtiva dos ovinos no Brasil: organização e comercialização. In: SIMPÓSIO DE OVINOCULTURA DE CORTE DE MARÍlIA, 1., 2007, Marília. Anais... Marília: Universidade de Marília, 2007. (CD-ROM)

VIANA, J.G.A. Governança da cadeia produtiva da ovinocultura no Rio Grande do Sul: estudo de caso à luz dos Custos de Transação e Produção. 116 p. Dissertação (Mestrado) Universidade Federal de Santa Maria, Centro de Ciências Rurais, Santa Maria. 2008.

VIANA, J.G.A., SILVEIRA, V.C.P. Análise econômica e custos de produção aplicados aos sistemas de produção de ovinos. In: CONGRESSO DA SOCIEDADE BRASILEIRA DE ECONOMIA, ADMINISTRAÇÃO E SOCIOLOGIA RURAL, 48., 2008, Rio Branco. Anais... Rio Branco: 2008a.

VIANA, J.G.A., SILVEIRA, V.C.P. Custos de produção e indicadores de desempenho: Metodologia aplicada a sistemas de produção de ovinos. Custos e agronegócio (online). 2008b, v. 4, n. 3, pp. 2-27.

WILLIAMSON, O. E. Transaction cost economics: the governance of contractual relations. The Journal of Law and Economics, v. 22, p. 233 - 261, 1979.

WILLIAMSON, O. E. The mechanisms of governance. Oxford University Press, New York, Oxford, 1996. 429 p.

YOKOYA, E.; PINHEIRO, J.V.; NAVES, J.R.; SILVA, M.R.; GAMEIRO, A.H. Estratégias de qualidade na produção de carne ovina: estudo de caso da VPJ Beef. IN: REUNIÃO ANUAL DA SOCIEDADE BRASILEIRA DE ZOOTECNIA, 46, 2009, Maringá. Anais... Maringá, 2009.

ZEOLA, N.M.B.L; SOBRINHO, A.G.S.; LEÃO, A.G.; PEREZ, H.L.; SANTOS, E.S. Homeopatia no controle de helmintos gastrintestinais de ovelhas em gestação. IN: REUNIÃO ANUAL DA SOCIEDADE BRASILEIRA DE ZOOTECNIA, 44, 2007, Jaboticabal. Anais... Jaboticabal, 2007. 